ORIENTAL JOURNAL OF
ISSN: 0974-6471
June 2017,
$\begin{aligned} & \text { COMPUTER SCIENCE \& TECHNOLOGY } \\ & \text { Pgs. No. (2): }\end{aligned}$
$\begin{gathered}\text { An International Open Free Access, Peer Reviewed Research Journal } \\ \text { Published By: Oriental Scientific Publishing Co., India. }\end{gathered}$
www.computerscijournal.org

\title{
Classification of Human Organ Using Image Processing
}

\author{
SINDHU* and V. VAIDHEHI \\ Department of Computer Sciences, Christ University, Bengaluru, India. \\ http://dx.doi.org/10.13005/ojcst/10.02.11
}

(Received: March 21, 2017; Accepted: May 20, 2017)

\begin{abstract}
The collection of large database of digital image has been used for efficient and advanced way for classifying and intelligent retrieval of medical imaging. This research work is to classify human organs based on MRI images. The various MRI images of organ have been considered as the data set. The main objective of this research work is to automate the medical imaging system. Digital images retrieved based on its shape by Canny Edge Detection and is clustered together in one class using K-Means Algorithm. 2564 data sets related to brain and heart is considered for this research work. The system was trained to classify the image which results in faster execution in medical field, also helped in obtain noiseless and efficient data.
\end{abstract}

Keyword: Medical imaging, digital images, organs, Canny Edge Detection, K-means Algorithm.

\section{INTRODUCTION}

Digital Image Processing is a technique of performing image processing on digital images using algorithms which categorise the digital signal in the input data. The base of DIP are mathematical and probabilistic formulas, but human intuition also plays the vital role in selecting the techniques based on the requirement. In medical imaging the body is scanned through magnetic resonance which helps in producing the minute details of human organs, tissues and bones. It helps in analysing the functionality of organs in human body. Medical imaging provides the visual impact of interior organs which helps in clinical diagnosis.
Imaging innovation in Medical Science made the specialists to analyse the inside bits of the body for simple determination. It likewise pushed specialists to make Nano surgeries for coming to the inside segments without truly opening excessively of the body parts. Computerized Tomography Scanner, Ultrasound and Attractive Resonance Imaging assumed control $\mathrm{x}$-beam imaging by making the specialists to take a gander at the body's tricky area and measuring the risk involved in it. With the CT Scanner, body's inside can be uncovered with ease and the unhealthy regions can be distinguished without bringing on either inconvenience or torment to the patient. X-ray gets signals from the body's attractive particles turning 
to its attractive tune and with the assistance of its effective system changes over scanner information into uncovering pictures of inner organs. Picture Handling methods created for breaking down remote detecting information might be altered to break down the yields of restorative imaging frameworks to get best-preferred standpoint to investigate side effects of the patients easily².

In this work the image retrieval is used which focus in extracting the properties related visualisation of an image than the textual properties. Image processing includes, processing based on various features which involves shape, texture, colour and dimension. This system involves the shape as essential feature for tokenizing the medical image database. This research include several processing steps, which are taking input as an image, extracting the features of image in the requirement of the system in this case shape is used as the primary feature, then classifying the image into one class and then storing the image into the database. These types of system are adding up to the necessities with the advancement of the technology. As medical database always deals with the complex data, so feature extraction should be performed carefully, later on it should be classified with an efficient techniques which helps in easy retrieval of the image. Medical image is always blended with high divergent data and variant structure ${ }^{8}$. The Watchful edge locator arranges a pixel as an edge if the slope greatness of the pixel is bigger than those of pixels at both its sides toward most extreme ends. In this paper we will demonstrate that characterizing edges in this way causes some undeniable edges to be missed. We will likewise demonstrate to re-examine the Shrewd edge finder to enhance its identification and exactness.

To identify this system various papers were referred on Digital Image Processing and its feature. This also helped in understanding the importance of Image processing and its requirement. In medical science Image processing plays an important role without performing the critical surgery. It shows the overview and helped the professional in meticulous planning that is required in surgery and its affect. It also gives an idea how the approach effect can be on patient. Image processing involves several steps to obtain the resultant image. The processed image is noiseless and efficient. Several paper explained the importance of canny edge deduction and the method of deducting the edges of the image. The filter is applied on the image to obtain clear view of the image, which later on will help in getting clear boundaries. The method of K-means algorithm helped in classifying in one class.

\section{Literature Review}

As the increasing demand of enhancement in medical field, faster execution and efficiency becomes the base criteria of any system ${ }^{1}$. Image processing is used to extract the feature of images. As medical data contain sensitive information, to resolve the complexity issue image processing is used, which in result will help in better diagnosing system. Image processing helps in extracting the feature and enhances the image.

Several areas in which image processing techniques are used in identifying the abnormalities or tumour in brain or breast ${ }^{2}$. Image processing techniques have created the major impact medical science. In this paper, we introduce a basic and productive execution of Lloyd's calculation, which we call the separating calculation. This calculation starts by putting away the information focuses in a kd-tree . In each phase of Lloyd's calculation, the closest focus to every information point is registered furthermore, ever time the middle pixel is moved to the centroid of the related neighbours. The main idea is to keep up for every hub of the tree and its subset . The contender for every hub are pruned, or "filtered" as they are proliferated to the hub's child. Since the kd-tree is figured for the information focuses for the centroid, there is no compelling reason to upgrade this structure with each phase of Lloyd's calculation. Moreover, since there are ordinarily numerous of larger number of information focuses on clustering of pixels, there are more prominent economies of scale to be figured it out. Take note of this is not another bunching strategy, but rather just an productive usage of Lloyd's k-implies calculation ${ }^{7}$.Grouping issues emerge in a wide range of utilizations, for example, information mining and learning disclosure, information pressure and vector quantization, and design acknowledgment and example order. The thought of what constitutes a decent bunch relies on upon the application and 
there are numerous strategies for discovering bunches subject to different criteria, both specially appointed and methodical. Bunching in light of k-means is firmly identified with a number of other bunching and area issues. These incorporate the Euclidean k-medians in which the goal is to limit the entirety of separations to the closest focus and the geometric the k-focus issue in which the goal is to limit the most extreme separation from each indicate its nearest focus.

Image processing applied to enhance the image and also helped in identifying the disease related to Lungs, histogram equalisation techniques are applied. The heartiness of surface examination makes it especially alluring for checking infection movement and in another words treatment reaction with respect to time, as it is exhibited with MS. Bolster Vector Machines utilizing the connection portion, Polynomial piece, Gaussian bit and RBF part all assume a critical part in every application and give acceptable comes about. Neural techniques are more helpful in consequently breaking down the pictures via preparing the systems. Information Mining methods are likewise assuming equivalent part in maintaining the image databases and taking examination on the arrangement of various pictures. According to Fluffy calculations which are giving better outcomes. Initial two cases are more muddled systems on account of its dreary computations, however giving great execution ${ }^{11}$. Arrangement alludes as to allocating the physical question or episode for the given arrangement of predefined classifications. Medicinal picture dataset which is utilised for picture characterization or for showing purposes regularly contain pictures of a wide range of modalities, brought under differed conditions with the variable exactness of comment. This can be valid for pictures found in different on-line assets, for those which are including to get into the on-line substance of diaries. Approaches joining the set of two visual and printed strategies for order have appeared some guarantee at therapeutic picture order assignments ${ }^{8}$.

In previous system it does not allow us to automatically classify the organs and group them in one class which increases possibility of human error. With the increasing there is an immediate need of system which can help in automating the identification process and can make our work faster. This system helps in taking image as an input, preprocess it by removing the noise and cropping it and the collecting it into one format. Then with the help of image processing it is enhanced and narrowed down to the data which needs to be processed. The enriched data are the one through which feature will be identified and will be extracted.

\section{METHODOLOGY}

\section{Dataset-MRI Images}

Various organs have been considered to classify the types of organs into various categories. The input dataset consist of all MRI images. We considered MRI for our dataset as it works on using magnetics fields and radio frequency pulses which helps in scanning the minute details of organs, tissues and all internal body structure. Generally, an MRI image provides more clear and differentiated images of the organs as compared with CT scan images. After collecting all the images, the images should be brought down in one category i.e. all the images should be in one format which is the base criteria of image processing.

In this work all the images is grey scale images, 2D-images as well as in .jpg format. In this work animated image and coloured images is not been considered, to avoid the complexity of the image.

\section{Approach \\ Pre-Processing}

MRI images can be affected to noise, which can be occurred during the transmission of images. The noises of the images have been reduced and the unwanted data has been cropped manually. Enhancement of data is necessary for the best possible result, which will help down in narrowing towards our goal.

\section{Feature Extraction}

In image processing all the images are classified under certain features which are texture, shape $^{3}$, colour. Feature extraction is an integral part of image processing which helps in narrowing down our dataset to achieve our goal. The identified feature is taken as the input in the system based on which the organs will be classified and stored 
in the database. In the gray scale images the gray levels helps in determining the pixel and its relevant position. By identifying the core pixel plotting the neighbouring pixels becomes more easier. In this system as shape is taken as primary feature which is also very important feature in identification, Canny Edge Detection ${ }^{4}$ helps in filtering out the edges of the medical images. In this system for every input image the shape has been determined for every organ, there are multiple images taken into consideration for identifying the edge of one organ to get good result.

\section{Classification}

Organ classification is last but important part of this system. After identifying the feature of your dataset, classification works as identifying the various regions or the edges of your images, so that a quicker and efficient result can be extracted from the voluminous database. In this work K-mean algorithm has been implemented for clustering the images based on its regions identified, and

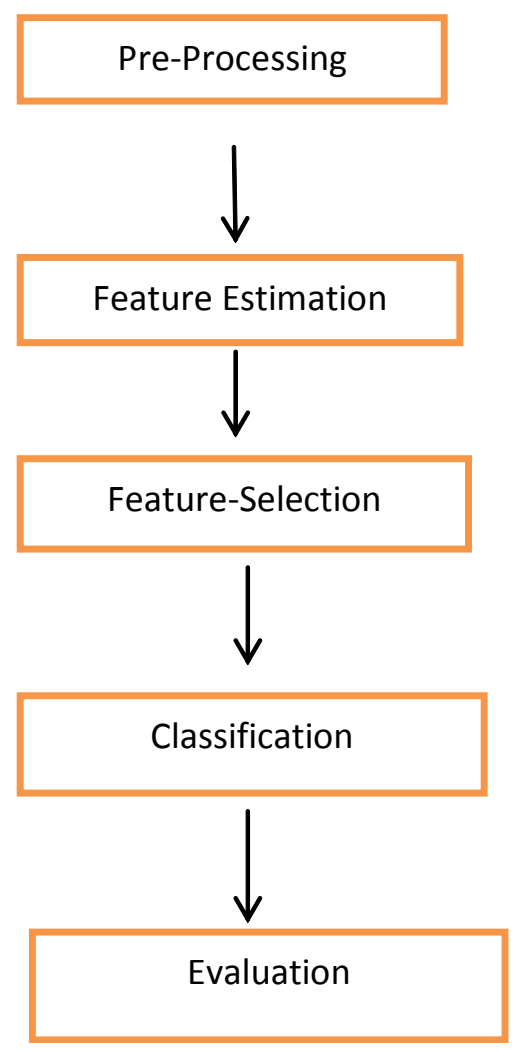

Fig. 1: Steps involved in Medical Image Processing considering them into a single class of a particular organ. K-mean algorithm works depending on the mean value of the entity present in the bundle. In this case, it the pixel of the medical image of which the mean value of the pixels will be taken. For each cluster a centroid is being initialised, and then the mean is determined for every cluster. Every centroid should be at certain distance from the other centroid. After every processing, new centroid should be initialised. When all the centroids have been determined the clustering is completed.

\section{RESULT AND DISCUSSIONS}

The work involved MRI images which pre-processed manually and narrowed down in one format. Various features were analysed while performing the image processing. Among which we considered shape as a powerful feature in identification of the organ. Edge detection and with the help of filter a proper segmented shape of organ is obtained. Every organ is classified into one

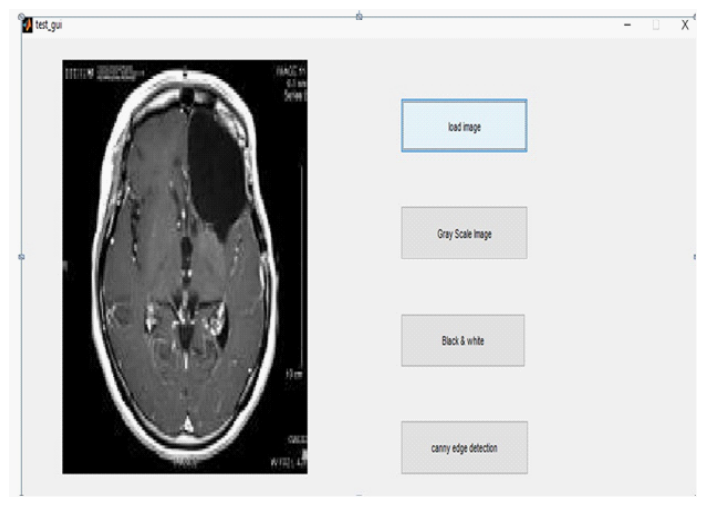

Fig. 1: Input Image of MRI Scan of brain

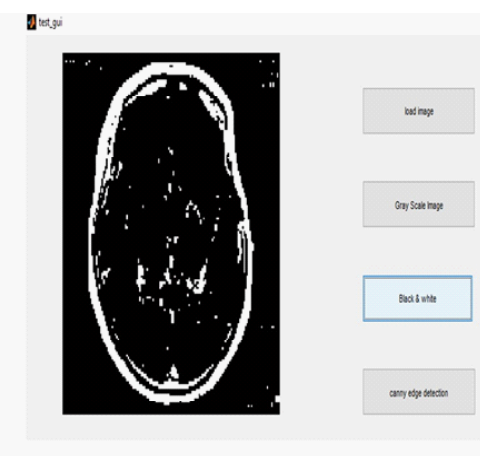

Fig. 2: Black And white image of Brain in Matlab 
category using K-means algorithm. It was tested with providing with the test image of various organs and classifying in one category.

\section{CONCLUSION}

In this paper, the medical image retrieval proposed in this system relies on the fetching of image based on its shape which is identify using Canny Edge Detection Algorithm, with the help of canny edge detection we are able to retrieve the border or the outer line of the scanned image and using filter method the noise from the processed image and K-means algorithm is used for clustering similar type of organs. Then matching the test image with the extracted image from the database.

This system will help in better diagnosing of the patients. Earlier the manual identification increases the chances of more human error, which in result risks the life of patients. With the improved system surgical planning as well as execution became faster and easier.
As this system has taken shape as base feature, it can combine all the feature of images such as colour, texture, shape, size, dimensions to give more accuracy to the result. As this work only contains $2 \mathrm{D}$ images and all the images are in grayscale, it can be modified for $3 \mathrm{D}$, coloured images. The system advancement can also be done by considering animated images and enhanced features of image processing.

\section{ACKNOWLEDGEMENT}

In writing this paper I got immense support of my guide Asst. Prof. Vaidhehi V for guiding me throughout the paper and Prof. Anitha HB and Prof. Ramurthy $\mathrm{B}$ for reviewing the paper and giving the constructive feedback. I would like to thanks my Head Of Department Prof Joy Poluse Computer Science Department, Christ University, Bengaluru for providing the useful resource and his guidance. I would like to thanks my Parents for providing the funding for this research paper.

\section{REFERENCES}

1. MEDICAL IMAGE PROCESSING K.M.M. Rao, V.D.P. Rao

2. Canny, J., "A computational approach to edge detection", IEEE Trans on Pattern Analysis and Machine Intelligence, 8:679698, 1986.

3. CBMIR: Shape-Based Image Retrieval Using Canny Edge Detection And K-mean Clustering Algorithm For Medical Images.B .Ramamurthy1,K.R.Chandran2

4. Diagnose Breast Cancer Through Mammograms, Using Image Processing Techniques and Optimization Techniques. Dr. M. Karnan1, K. Rajiv Gandhi2.

5. An Efficient k-Means Clustering Algorithm: Analysis and Implementation. Tapas Kanungo, Senior Member, IEEE, David M. Mount, Member, IEEE, Nathan S. Netanyahu, Member, IEEE, Christine D. Piatko, Ruth Silverman, and Angela Y. Wu, Senior Member, IEEE. IEEE Transactions On Pattern Analysis And Machine Intelligence, 24(7), JULY 2002.

6. http://www.ijcaonline.org/icvci/number11/ icvci1458.pdf
7. http://www.ace.tuiasi.ro/users/103/ f2_2011_7_(83-98)_Smochina.pdf

8. A Review of Medical Image Classification Techniques. Smitha P. Selection grade lecturer Dept. of CSE, CE Karunagapally Shaji L. Lecture in comp.Appln Dept. of IT, CE, Karunagapally Dr. Mini M. G. Asst. Prof. \& HOD Dept. of ECE, MEC Karunagapally International Conference on VLSI, Communication \& Instrumentation (ICVCI) 2011 Proceedings published by International Journal of Computer Applications(IJCA)

9. Thies C, Guld MO, Fischer B, Lehmann TM, "Content- based queries on the Caslmage database with in the IRMA framework", Lec Notes in Comp Sci; 3491:781-92. 2005.

10. Thoma GR, Long LR, Antani SK, Biomedical Imaging research and development: knowledge from images in the medical enterprise. Technical Report Lister Hill National Ctr for Biomedical Communications, US National Library of Medicine, NIH 2006;:LHNCBCTR-2006-002. 